Supplement of Biogeosciences, 12, 4209-4220, 2015

http://www.biogeosciences.net/12/4209/2015/

doi:10.5194/bg-12-4209-2015-supplement

(C) Author(s) 2015. CC Attribution 3.0 License.

(c) (1)

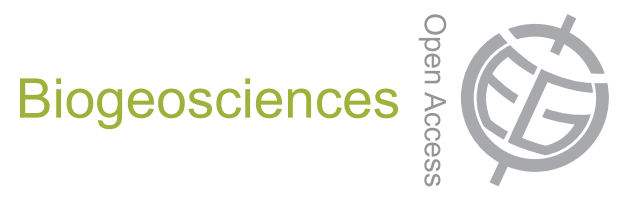

Supplement of

\title{
Impact of seawater carbonate chemistry on the calcification of marine bivalves
}

\section{J. Thomsen et al.}

Correspondence to: J. Thomsen (jothomsen@ucsd.edu)

The copyright of individual parts of the supplement might differ from the CC-BY 3.0 licence. 
Table S1 Meta-analysis of larval calcification responses expressed as \% of control shell length and the corresponding carbonate chemistry.

\begin{tabular}{|c|c|c|c|c|c|c|c|c|c|c|}
\hline $\begin{array}{c}{\left[C_{\mathrm{T}}\right]} \\
(\mu \mathrm{mol} \mathrm{kg}) \\
\end{array}$ & $\begin{array}{c}\mathrm{pH} \\
\text { total } \\
\text { scale } \\
\end{array}$ & $\begin{array}{c}{\left[\mathrm{HCO}_{3}{ }^{-}\right]} \\
(\mu \mathrm{mol} \\
\left.\mathrm{kg}^{-1}\right)\end{array}$ & $\begin{array}{c}p \mathrm{CO}_{2} \\
{[\mu \mathrm{atm}]}\end{array}$ & $\begin{array}{c}{\left[\mathrm{CO}_{3}{ }^{2-}\right]} \\
\left(\mu \mathrm{mol} \mathrm{kg}{ }^{-1}\right)\end{array}$ & Saragonite & $\begin{array}{c}{\left[C_{\mathrm{T}}\right] /\left[\mathrm{H}^{+}\right]} \\
{[\mathrm{mol}] /[\mu \mathrm{mol}]}\end{array}$ & $\begin{array}{l}{\left[\mathrm{HCO}_{3}{ }^{-}\right] /\left[\mathrm{H}^{+}\right]} \\
{[\mathrm{mol}] /[\mu \mathrm{mol}]}\end{array}$ & $\begin{array}{l}\text { shell length } \\
\% \text { of control }\end{array}$ & reference & species \\
\hline 1863 & 7.99 & 1766 & 508 & 78 & 1.26 & 0.18 & 0.174 & 100 & this study & Mytilus edul \\
\hline 2033 & 7.49 & 1937 & 1777 & 27 & 0.43 & 0.06 & 0.060 & 79 & & \\
\hline 848 & 7.63 & 811 & 539 & 15 & 0.25 & 0.04 & 0.035 & 79 & & \\
\hline 3399 & 7.72 & 3254 & 1770 & 76 & 1.23 & 0.18 & 0.170 & 99 & & \\
\hline 2839 & 8.16 & 2583 & 430 & 240 & 3.76 & 0.41 & 0.372 & 101 & this study & M. edulis \\
\hline 2463 & 8.08 & 2269 & 449 & 177 & 2.78 & 0.30 & 0.275 & 95 & & \\
\hline 1996 & 7.99 & 1862 & 460 & 116 & 1.83 & 0.19 & 0.181 & 100 & & \\
\hline 1583 & 7.91 & 1488 & 440 & 78 & 1.22 & 0.13 & 0.121 & 101 & & \\
\hline 1101 & 7.78 & 1044 & 415 & 41 & 0.64 & 0.07 & 0.063 & 87 & & \\
\hline 741 & 7.59 & 707 & 436 & 18 & 0.28 & 0.03 & 0.028 & 54 & & \\
\hline 6283 & 7.70 & 5980 & 2859 & 193 & 3.03 & 0.32 & 0.300 & 85 & & \\
\hline 5525 & 7.67 & 5263 & 2712 & 158 & 2.48 & 0.26 & 0.245 & 94 & & \\
\hline 4589 & 7.58 & 4376 & 2774 & 107 & 1.67 & 0.17 & 0.166 & 93 & & \\
\hline 3553 & 7.45 & 3382 & 2855 & 62 & 0.97 & 0.10 & 0.096 & 66 & & \\
\hline 1490 & 7.10 & 1378 & 2631 & 11 & 0.17 & 0.02 & 0.017 & 62 & & \\
\hline 2271 & 8.03 & 2090 & 468 & 164 & 2.56 & 0.24 & 0.222 & 100 & $\begin{array}{l}\text { Gazeau et } \\
\text { al. } 2010\end{array}$ & M. edulis \\
\hline 2408 & 7.69 & 2285 & 1123 & 82 & 1.28 & 0.12 & 0.111 & 95 & & \\
\hline 2253 & 7.97 & 2091 & 537 & 143 & 2.22 & 0.21 & 0.194 & 99 & & \\
\hline 2439 & 7.46 & 2320 & 1930 & 48 & 0.75 & 0.07 & 0.066 & 87 & & \\
\hline 2232 & 7.90 & 2076 & 639 & 135 & 2.13 & 0.18 & 0.164 & 97 & & \\
\hline 1813 & 8.17 & 1644 & 254 & 159 & 2.42 & 0.27 & 0.243 & 100 & $\begin{array}{l}\text { Sunday et al. } \\
2011\end{array}$ & M. trossulus \\
\hline 1937 & 7.86 & 1826 & 571 & 87 & 1.33 & 0.14 & 0.133 & 97 & & \\
\hline 2021 & 8.04 & 1851 & 399 & 155 & 2.40 & 0.22 & 0.203 & 100 & $\begin{array}{l}\text { Frieder et al. } \\
2014\end{array}$ & M. california \\
\hline 2214 & 7.51 & 2106 & 1538 & 52 & 0.81 & 0.07 & 0.068 & 91 & & \\
\hline 2174 & 7.64 & 2065 & 1118 & 69 & 1.07 & 0.09 & 0.090 & 94 & & \\
\hline 2039 & 8.00 & 1880 & 444 & 144 & 2.22 & 0.20 & 0.188 & 100 & & \\
\hline 2082 & 7.90 & 1943 & 578 & 118 & 1.83 & 0.17 & 0.154 & 100 & & \\
\hline 2161 & 7.68 & 2050 & 1012 & 75 & 1.16 & 0.10 & 0.098 & 98 & & \\
\hline 1780 & 8.02 & 1645 & 360 & 121 & 1.83 & 0.19 & 0.172 & 100 & $\begin{array}{l}\text { Kurihara et } \\
\text { al. } 2009\end{array}$ & $\begin{array}{l}\text { M. } \\
\text { galloprovinc }\end{array}$ \\
\hline 1983 & 7.31 & 1873 & 2104 & 27 & 0.41 & 0.04 & 0.038 & 69 & & \\
\hline 2850 & 8.10 & 2574 & 482 & 259 & 3.99 & 0.36 & 0.324 & 100 & $\begin{array}{l}\text { Vihtakari et } \\
\text { al. } 2013\end{array}$ & $\begin{array}{l}\text { M. } \\
\text { gallopovinici }\end{array}$ \\
\hline 2859 & 7.73 & 2701 & 1185 & 116 & 1.79 & 0.15 & 0.145 & 96 & & \\
\hline 2061 & 7.95 & 1913 & 507 & 130 & 2.02 & 0.18 & 0.170 & 100 & $\begin{array}{l}\text { Frieder et al. } \\
2014\end{array}$ & $\begin{array}{l}\text { M. } \\
\text { gallopovinici }\end{array}$ \\
\hline 2205 & 7.54 & 2098 & 1430 & 56 & 0.86 & 0.08 & 0.073 & 92 & & \\
\hline 2199 & 7.56 & 2091 & 1361 & 58 & 0.90 & 0.08 & 0.076 & 93 & & \\
\hline 2078 & 7.91 & 1937 & 563 & 120 & 1.86 & 0.17 & 0.157 & 100 & & \\
\hline 2183 & 7.61 & 2075 & 1204 & 65 & 1.00 & 0.09 & 0.085 & 95 & & \\
\hline 2061 & 7.95 & 1913 & 507 & 130 & 2.02 & 0.18 & 0.170 & 100 & & \\
\hline 2189 & 7.59 & 2082 & 1265 & 62 & 0.96 & 0.09 & 0.081 & 96 & & \\
\hline 1703 & 8.08 & 1517 & 314 & 177 & 2.80 & 0.20 & 0.181 & 100 & $\begin{array}{l}\text { Kurihara et } \\
\text { al. } 2007\end{array}$ & $\begin{array}{l}\text { Crassostrea } \\
\text { gigas }\end{array}$ \\
\hline
\end{tabular}




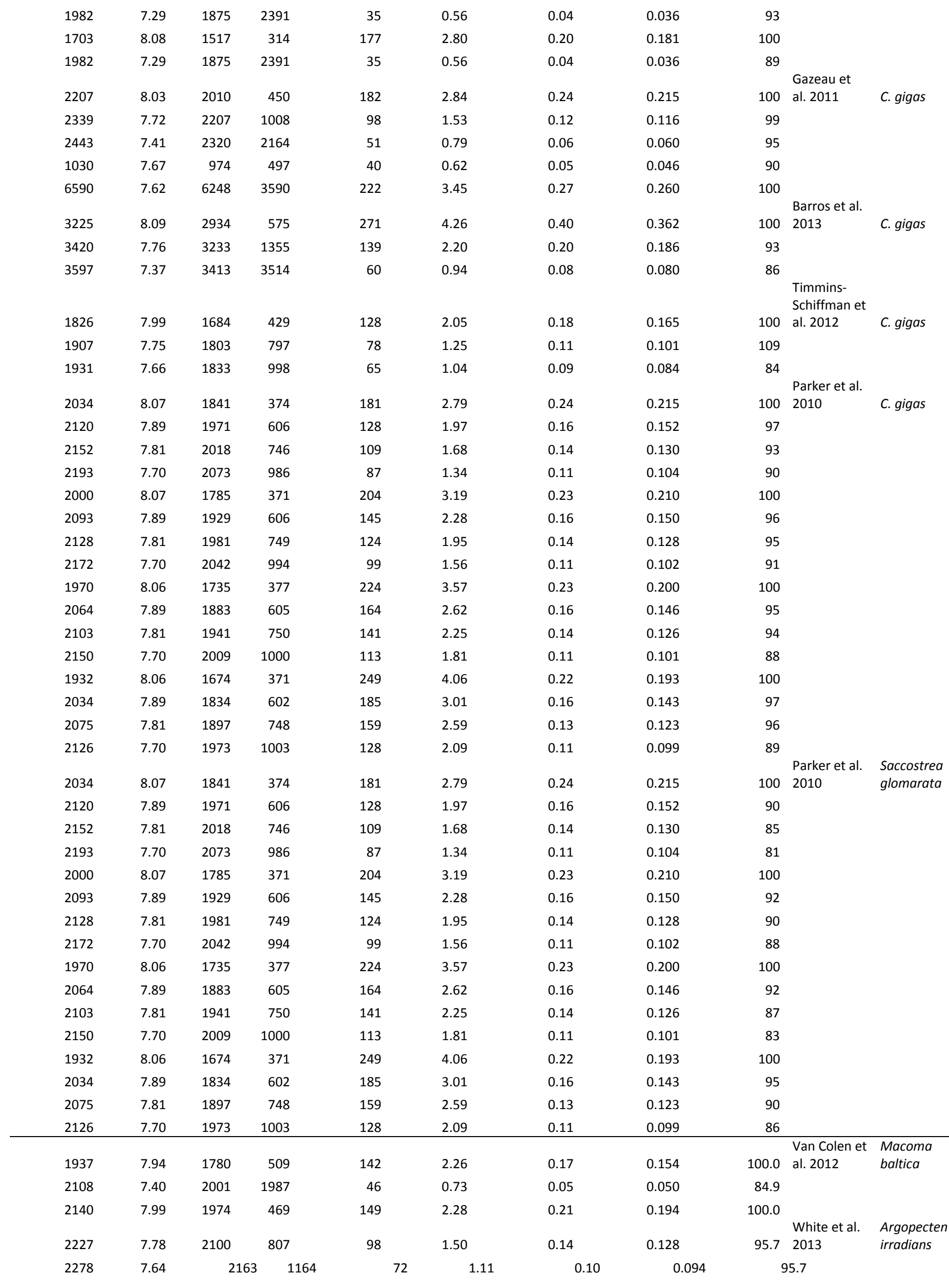




\begin{tabular}{rrrrrrrrrr}
2320 & 7.51 & 2207 & 1599 & 54 & 0.83 & 0.07 & 0.071 & 91.3 & Andersen et al. 2013 \\
2781 & 8.00 & 2604 & 637 & 152 & 2.39 & 0.28 & 0.260 & 0.126 \\
2914 & 7.66 & 2781 & 1498 & 73 & 1.15 & 0.13 & 9.7 \\
3001 & 7.42 & 2853 & 2640 & 44 & 0.69 & 0.08 & 0.075 \\
\hline
\end{tabular}

Table S2 Meta-analysis of juvenile calcification responses expressed as \% of control shell mass growth and the corresponding carbonate chemistry.

\begin{tabular}{|c|c|c|c|c|c|c|c|c|}
\hline $\begin{array}{c}{\left[C_{\mathrm{T}}\right]} \\
(\mu \mathrm{mol} \mathrm{kg})\end{array}$ & $\begin{array}{c}\mathrm{pH} \\
\text { total scale }\end{array}$ & $\begin{array}{c}{\left[\mathrm{HCO}_{3}^{-}\right]} \\
\left(\mu \mathrm{mol} \mathrm{kg}{ }^{-1}\right)\end{array}$ & $\begin{array}{c}p \mathrm{CO}_{2} \\
{[\mu \mathrm{atm}]}\end{array}$ & $\begin{array}{c}{\left[\mathrm{CO}_{3}{ }^{2-}\right]} \\
\left(\mu \mathrm{mol} \mathrm{kg}{ }^{-1}\right)\end{array}$ & Saragonite & $\begin{array}{c}{\left[C_{\mathrm{T}}\right] /\left[\mathrm{H}^{+}\right]} \\
{[\mathrm{mol}] /[\mu \mathrm{mol}]}\end{array}$ & $\begin{array}{c}{\left[\mathrm{HCO}_{3}^{-}\right] /\left[\mathrm{H}^{+}\right]} \\
{[\mathrm{mol}] /[\mu \mathrm{mol}]}\end{array}$ & $\begin{array}{c}\text { shell mass growth } \\
\% \text { of control }\end{array}$ \\
\hline 1888 & 8.13 & 1783 & 501 & 82 & 1.32 & 0.25 & 0.24 & 100 this study \\
\hline 2246 & 7.16 & 2035 & 5214 & 10 & 0.16 & 0.03 & 0.03 & 66 \\
\hline 847 & 7.74 & 809 & 564 & 15 & 0.24 & 0.05 & 0.04 & 50 \\
\hline 5909 & 7.57 & 5616 & 5649 & 71 & 1.14 & 0.22 & 0.21 & 106 \\
\hline 1891 & 8.01 & 1797 & 479 & 73 & 1.14 & 0.19 & 0.18 & 100 Thomsen et al. 2010 \\
\hline 1984 & 7.60 & 1898 & 1287 & 30 & 0.48 & 0.08 & 0.08 & 97 \\
\hline 2126 & 7.13 & 1946 & 3912 & 11 & 0.17 & 0.03 & 0.03 & 65 \\
\hline 1891 & 8.01 & 1797 & 479 & 73 & 1.14 & 0.19 & 0.18 & 100 \\
\hline 1984 & 7.60 & 1898 & 1287 & 30 & 0.48 & 0.08 & 0.08 & 91 \\
\hline 2126 & 7.13 & 1946 & 3912 & 11 & 0.17 & 0.03 & 0.03 & 56 \\
\hline 1969 & 8.02 & 1874 & 449 & 73 & 1.11 & 0.21 & 0.20 & 100 Thomsen and Melzner 20 \\
\hline 2031 & 7.74 & 1947 & 886 & 40 & 0.61 & 0.11 & 0.11 & 90 \\
\hline 2124 & 7.41 & 2007 & 1978 & 19 & 0.30 & 0.05 & 0.05 & 77 \\
\hline 2214 & 7.14 & 2020 & 3685 & 10 & 0.16 & 0.03 & 0.03 & 64 \\
\hline 1882 & 7.95 & 1806 & 508 & 44 & 0.72 & 0.17 & 0.16 & 100 Melzner et al. 2011 \\
\hline 1952 & 7.61 & 1863 & 1138 & 21 & 0.34 & 0.08 & 0.08 & 103 \\
\hline 2033 & 7.26 & 1875 & 2540 & 10 & 0.15 & 0.04 & 0.03 & 57 \\
\hline 2097 & 7.08 & 1865 & 3859 & 6 & 0.10 & 0.03 & 0.02 & 63 \\
\hline 1775 & 8.01 & 1680 & 460 & 77 & 1.29 & 0.18 & 0.17 & 100 Thomsen et al. 2013 \\
\hline 1878 & 7.70 & 1798 & 1006 & 40 & 0.67 & 0.09 & 0.09 & 76 \\
\hline 1964 & 7.40 & 1862 & 2078 & 21 & 0.35 & 0.05 & 0.05 & 75 \\
\hline 2035 & 7.19 & 1888 & 3417 & 13 & 0.23 & 0.03 & 0.03 & 58 \\
\hline
\end{tabular}

Fig. S1. Meta-analysis of the calcification response (measured as shell length) in \% of control of bivalve larvae larvae kept under modified carbonate chemistry during the lecitotrophic phase. Calcification response is plotted against seawater (A) pCO2, (B) [HCO3-], (C) $\left[\mathrm{CO}_{3}{ }^{2-}\right]$ and (D) $\mathrm{pH}$. Regressions depict an exponential rise to maximum: $f=y 0+a^{*}\left(1-\exp \left(-b^{*} x\right)\right)$.

Fig. S2. Delay of shell formation in M. edulis larvae two days after fertilization.

Carbonate chemistry corresponds to the treatments (pCO2/[ $\left.\mathrm{CO}_{3}{ }^{2-}\right]$ ) given in Table 1: (A) 390/78, B) 2400/20, C) 390/20, D) 2400/78. 
Fig. S1
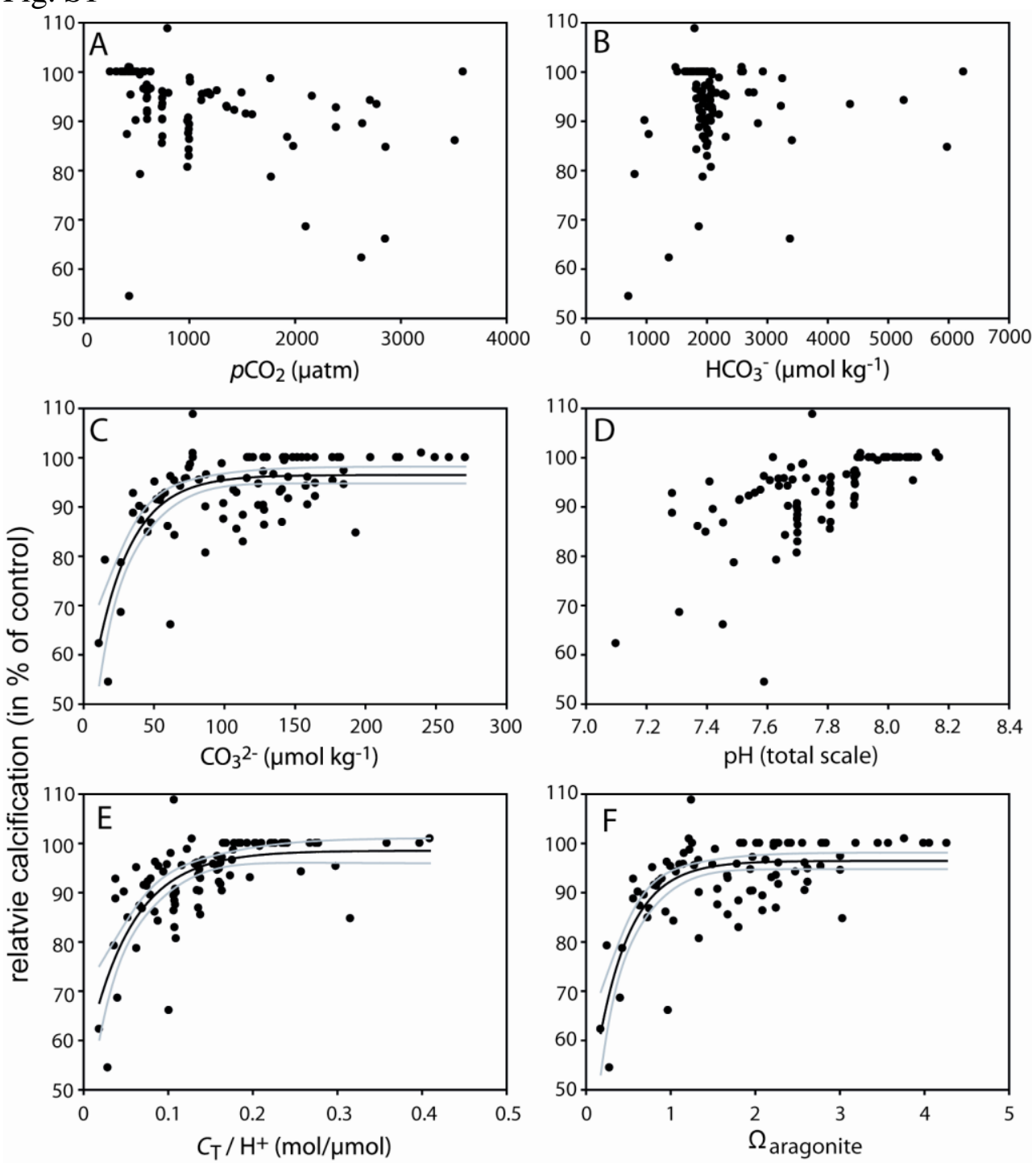

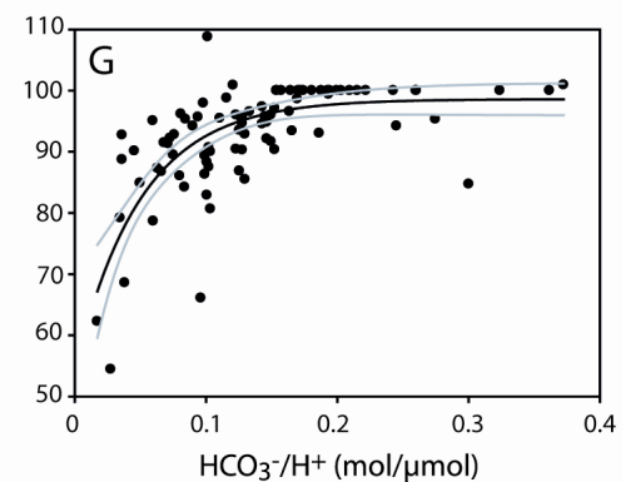


Fig. S2
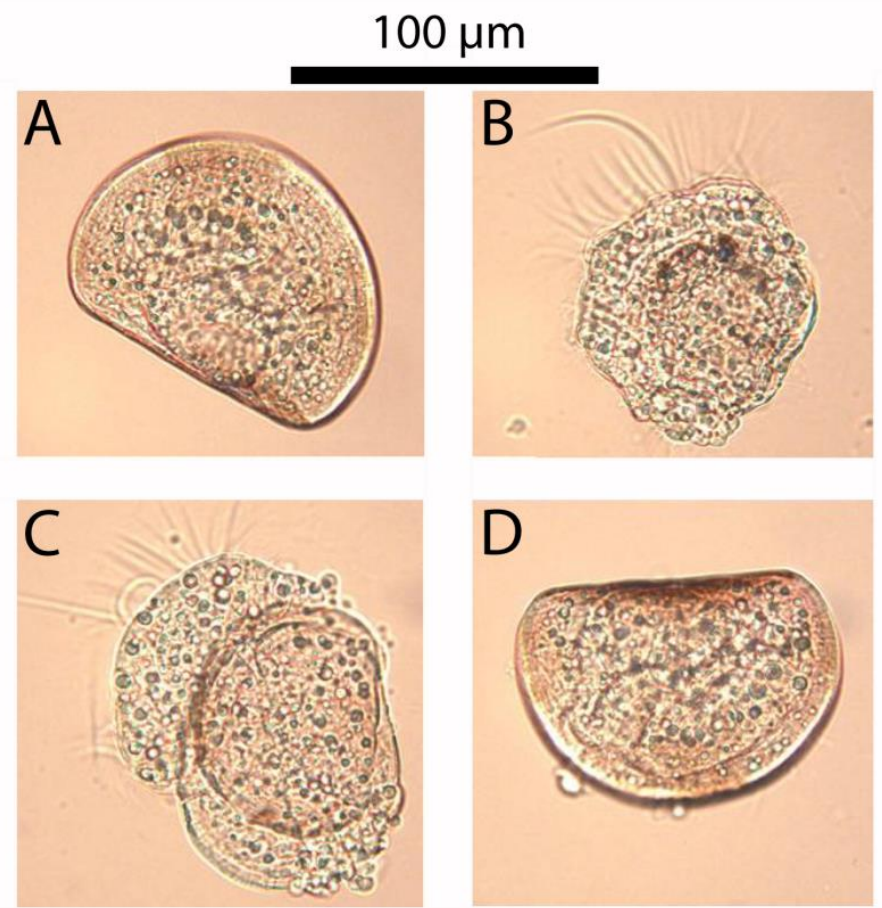